\title{
Differential Expression of Hyperpolarization-Activated Currents Reveals Distinct Classes of Visual Cortical Projection Neurons
}

\author{
Joel S. Solomon, ${ }^{1}$ John F. Doyle, ${ }^{1}$ Andreas Burkhalter, ${ }^{2}$ and Jeanne M. Nerbonne ${ }^{1}$ \\ Departments of 'Molecular Biology and Pharmacology and ${ }^{2}$ Neurosurgery, Washington University School of Medicine, St. \\ Louis, Missouri 63110
}

\begin{abstract}
Combining in vivo retrograde labeling and in vitro electrophysiological recording techniques, we examined the distributions, densities, and biophysical properties of hyperpolarization-activated inward currents in two types of isolated, identified visual cortical projection neurons, superior colliculus-projecting (SCP) and callosal-projecting (CP) cells. In SCP cells, two kinetically distinct time-dependent hyperpolarization-activated inward current components are present. We have termed these $I_{h, l}$ and $I_{h, s}$ to denote the fast and slow components, respectively, of $I_{n}$ activation. In CP cells, in contrast, $I_{h, t}$ and $I_{h, s}$ are differentially expressed. In $59 \%$ of the CP cells examined, for example, both $I_{h, l}$ and $I_{h . s}$ were present. The properties of the currents are indistinguishable from those recorded from SCP cells, although both $I_{h, r}$ and $I_{h, s}$ are expressed at significantly lower densities in this subset of CP cells (as compared to the current densities in SCP cells). Of the remaining $41 \%$ of the CP cells studied, $26 \%$ were found to express only $I_{h, s}$ and $12 \%$ of the cells expressed neither $l_{h, t}$ nor $l_{h, s}$. Taken together, these results reveal that the electrical properties of $\mathrm{CP}$ visual cortical neurons are considerably more heterogeneous than those of SCP cells. The differential expression of $I_{h, t}$ and $I_{h, s}$ is expected to influence the integrated responses of different types of cortical projection neurons to excitatory and inhibitory synaptic inputs.
\end{abstract}

[Key words: identified neurons, patch clamp, voltage clamp, postinhibitory excitation, fluorescent beads]

Although analysis of the mammalian cortex has traditionally emphasized connectivity patterns to explain neuronal response properties, it seems certain that information processing in complex cortical circuits depends not only on the synaptic arrangement of the constituent neuronal elements, but also on how signals are transformed by these elements. Intracellular recordings in vivo and in vitro suggest that the intrinsic membrane properties of different types of cortical neurons are distinct. For example, cortical neurons have been classified as "regular spik-

\footnotetext{
Received Jan. 26, 1993; revised June 7, 1993; accepted June 10, 1993.

We thank Dr. Chris Lingle for providing us with BINFirs. We are also most grateful to Drs. Chris Lingle, Joe Henry Steinbach, and Bob Wilkinson for many helpful discussions throughout the course of this work. Finally, we acknowledge the financial support provided by the National Science Foundation (BNS-8809823) and the National Institutes of Health (T32-GM07205 and T32-GM07200).

Correspondence should be addressed to Dr. Jeanne M. Nerbonne, Department of Molecular Biology and Pharmacology, Washington University School of Medicine, 660 South Euclid Avenue, Box 8103, St. Louis, MO 63110.

Copyright (C) 1993 Society for Neuroscience $0270-6474 / 93 / 135082-10 \$ 05.00 / 0$
}

ing," "fast spiking," or "bursting" based on stereotyped differences in action potential waveforms and repetitive firing properties (McCormick et al., 1985; Connors and Gutnick, 1990). Cortical neurons have also been classified according to distinct subthreshold electrical response properties (Mason and Larkman, 1990) and, more recently, according to the density of voltage-gated ion channels (Giffin et al., 1991). Interestingly, available evidence suggests that the observed electrophysiological distinctions among cortical neurons correlate with specific anatomical or biochemical features of the cells or with the circuits in which the cell participate (Connors and Gutnick, 1990). For example, some layer 5 cells are bursting, whereas others are regular spiking (Chagnac-Amitai et al., 1990; Connors and Gutnick, 1990; Mason and Larkman, 1990), and the time-dependent responses to cell hyperpolarization in layer 5 bursting and regular spiking cells are distinct (Mason and Larkman, 1990).

Assuming that the finding of electrophysiological phenotypes must reflect differences in the expression of distinct membrane conductance pathways in different cortical cell types, we have developed methods that allow us to identify different types of cortical neurons in vitro and to examine the membrane properties of these cells directly (Giffin et al., 1991; Solomon and Nerbonne, 1993a,b). In the experiments described here, we have combined whole-cell recording with in vivo retrograde labeling techniques and examined the hyperpolarization-activated currents in two types of visual cortical projection neurons, superior colliculus-projecting (SCP) and callosal-projecting (CP) cells. SCP neurons are a homogeneous population of large, layer 5 pyramidal cells with a prominent apical dendrite that extends to layer 1, where it ends in a characteristic tuft (Schofield et al., 1987; Hallman et al., 1988). Intracellular recordings from layer 5 neurons with this morphology suggest that SCP cells are bursting cells (Chagnac-Amitai et al., 1990; Mason and Larkman, 1990). CP neurons, in contrast, are anatomically (Ivy et al., 1979; Innocenti, 1981), morphologically (Voigt et al., 1988; Buhl and Singer, 1989), and physiologically (Harvey, 1980) heterogeneous. Nevertheless, CP neurons are expected to be regular spiking cells (Connors and Gutnick, 1990; Mason and Larkman, 1990).

Recently, we reported the presence of a hypcrpolarizationactivated inward current, termed $I_{h}$, that underlies the slow depolarizing sag (Mason and Larkman, 1990) in membrane potential observed in SCP cells during maintained hyperpolarizing current injections (Solomon and Nerbonne, 1993a). In addition, we demonstrated that deactivation of $I_{h}$ is responsible for a transient overshoot (Mason and Larkman, 1990) of the membrane potential following the offset of a prolonged hyper- 
polarizing input (Solomon and Nerbonne, 1993a). Similar to other "nontraditional inwardly rectifying" currents (DiFrancesco, 1981), $I_{h}$ in SCP cells is a mixed sodium and potassium current that begins to activate near $-60 \mathrm{mV}$ (Solomon and Nerbonne, 1993a). Unlike other cells, however, $I_{h}$ in visual cortical cells comprises two kinetically distinct components, which we have termed $I_{h . f}$ and $I_{h, s}$ to denote the fast and slow components, respectively, of inward current activation (Solomon and Nerbonne, 1993b). Here, we demonstrate that $I_{h, j}$ and $I_{h . s}$ are differentially distributed in SCP and CP cells, thereby revealing electrophysiologically distinct classes of cortical projection neurons.

\section{Materials and Methods}

Cell identification. Whole-cell voltage-clamp recordings were obtained from identified superior colliculus-projecting (SCP) and callosal-projecting $(\mathrm{CP})$ neurons isolated from rat primary visual cortex on postnatal day 9 (P9), P12, and P15, and maintained for varying times in vitro up to $2.5 \mathrm{~d}$. Methods for identifying, isolating, and recording from cortical projection neurons were essentially identical to those previously described (Giffin et al., 1991; Solomon and Nerbonne 1993a,b).

Projection neurons were identified in vitro following in vivo retrograde labeling with fluorescent microspheres (Lumafluor). SCP cells were labeled by pressure injection of rhodamine beads (Katz et al., 1984) into the ipsilateral superior colliculus of P5-P6 Long-Evans rat pups (Huettner and Baughman, 1986; Thong and Dreher, 1986) under halothane anaesthesia. CP cells were labeled at the same time by pressure injection of fluorescein beads (Katz and Iarovici, 1990) into the contralateral primary visual cortex. On P9, P12, or P15, labeled primary visual cortex was isolated and dissociated using a modified version (Giffin et al., 1991) of the procedure of Huettner and Baughman (1986). Isolated visual cortical neurons were plated on previously prepared monolayers of cortical astrocytes (Raff et al., 1979) and maintained in a humidified 95\% $\mathrm{O}_{2}, 5 \% \mathrm{CO}_{2}$ atmosphere at $37^{\circ} \mathrm{C}$. Isolated SCP and $\mathrm{CP}$ neurons could be easily identified in vitro under epifluorescence illumination at $400 \times$ (Fig. 1).

In selected animals, SCP and CP cells were examined in slide-mounted coronal sections through primary visual cortex. Following deep anesthesia with phenobarbital, P13 rat pups were perfused transcardially with $4 \%$ paraformaldehyde in $0.1 \mathrm{M}$ phosphate buffer. The brains were removed and incubated for $2 \mathrm{~d}$ in $4 \%$ paraformaldehyde containing $30 \%$ sucrose. Fifty micrometer coronal sections through primary visual cortex were then cut on a freezing microtome, mounted on gelatin-coated glass slides, and coverslipped. Alternate Nissl (cresyl violet)-stained sections were used to demarcate the boundaries of area 17 and to delimit cortical laminae.

Electrophysiology. Whole-cell recordings (Hamill et al., 1981) were obtained from identified projection neurons at room temperature $\left(21^{\circ} \mathrm{C}\right)$. The bath solution contained (in $\mathrm{mm}$ ) $115 \mathrm{NaCl}, 25$ tetraethylammonium chloride, $10 \mathrm{~N}$-2-hydroxyethylpiperazine- $N^{\prime}$-2-ethanesulfonic acid (HEPES), 5 glucose, $2.5 \mathrm{CaCl}_{2}, 2 \mathrm{MgCl}_{2}$, and 0.001 tetrodotoxin. The recording pipette solution contained (in $\mathrm{mm}$ ) $140 \mathrm{KCl}, 10$ ethylene glycol-bis-(aminoethyl ether)- $N, N, N^{\prime}, N^{\prime}$, tetra-acetic acid (EGTA), 10 HEPES, 5 glucose, $3 \mathrm{Mg}$-ATP, $2.07 \mathrm{CaCl}_{2}$, and $0.5 \mathrm{Na}$-GTP. The free calcium concentration of this solution, measured with a calcium-sensitive electrode (Orion), was $100 \mathrm{~nm}$. The bath and pipette solutions were adjusted to a pH of 7.3 by the addition of concentrated $\mathrm{NaOH}$, $\mathrm{KOH}$, or Tris(hydroxymethyl)aminomethane (Tris base).

Current signals, obtained with a Dagan 8900 patch-clamp amplifier with $1 \mathrm{G} \Omega$ feedback resistor were low-pass filtered at $2 \mathrm{kHz}$ using an eight-pole Bessel filter (Frequency Devices) prior to digitization using a TL-1 interface (Axon Instruments) and storage on an IBM-AT computer. Voltage-clamp paradigms and data acquisition were controlled using pCLAMP (Axon Instruments). Adequacy of cell membrane voltage control was examined by fitting the decay phases of the capacitance transients recorded during $\pm 10 \mathrm{mV}$ voltage steps from a holding potential of $-60 \mathrm{mV}$ (Solomon and Nerbonne, 1993a). Only cells with capacitance transients well described by a single exponential, that is, the amplitude of any additional component was $\leq 3 \%$ of the amplitude of the primary exponential component, were considered to behave as a single electrical compartment, and only these cells were accepted for further analyses (see Table 1). The 3\% criterion was established because
Table 1. Cortical neurons analyzed as a function of age at isolation and time in culture

\begin{tabular}{|c|c|c|c|c|}
\hline \multirow[b]{2}{*}{ Cell type } & \multirow{2}{*}{$\begin{array}{l}\text { Post- } \\
\text { natal } \\
\text { age at } \\
\text { isola- } \\
\text { tion }\end{array}$} & \multicolumn{3}{|c|}{ Number of cells } \\
\hline & & Day 0 & Day 1 & Day 2 \\
\hline \multirow[t]{4}{*}{ Colliculus-projecting } & P9 & $11(92 \%)$ & $2(18 \%)$ & $3(20 \%)$ \\
\hline & P12 & $7(100 \%)$ & $11(83 \%)$ & $3(27 \%)$ \\
\hline & P15 & $4(100 \%)$ & $4(100 \%)$ & 0 \\
\hline & Total & $22(96 \%)$ & $17(61 \%)$ & $6(21 \%)$ \\
\hline \multirow{4}{*}{ Callosal-projecting } & p9 & $9(100 \%)$ & $8(89 \%)$ & $8(57 \%)$ \\
\hline & P12 & $8(100 \%)$ & $7(50 \%)$ & $6(33 \%)$ \\
\hline & P15 & $3(75 \%)$ & $4(100 \%)$ & $1(33 \%)$ \\
\hline & Total & $20(95 \%)$ & $19(70 \%)$ & $15(43 \%)$ \\
\hline
\end{tabular}

The numbers in parentheses are the percentages of cells examined at each time point that satisfied the selection criteria for adequate voltage control (see Materials and Methods).

preliminary analyses revealed that if the amplitude of a second exponential component was $\leq 3 \%$ of the first, the data were equally well fitted by one or two exponentials.

Data analysis. Data analysis was performed using pCLAMP, BINFITs (provided by Dr. Christopher Lingle, Washington University Medical School), and css STATISTICA (StatSoft). Time constants for inward current activation were determined by fitting Equation 1 to the data (see Results). In 14 of the 83 cells analyzed, the time constants for $I_{h, s}$, determined from fitted curves, were $>5 \mathrm{sec}$; in one of these 14 cells, the activation time constant for $I_{h, s}$ was $>50 \mathrm{~min}$. Because the hyperpolarization-activated current records from which these values were derived were only $4.4 \mathrm{sec}$ in duration, time constants $>5 \mathrm{sec}$ are considered meaningless. Therefore, all time constants greater than the arbitrary value of $5.3 \mathrm{sec}$ were ignored. Fitted current amplitudes $\leq 0.2 \mathrm{pA}$ were considered to be equal to zero. The statistical tests used to examine differences among cells are indicated throughout the text. Parametric statistical tests (i.e., $t$ test, ANOVA) were used when comparing population samples that were normally distributed. Otherwise, nonparametric tests, such as the Kolmogorov-Smirnov test or the KruskalWallis ANOVA by ranks, were performed. In all cases, statistical significance was set at the $p=0.05$ level.

\section{Results}

\section{Identification of cortical projection neurons}

Retrograde labeling techniques were used to identify SCP and $\mathrm{CP}$ neurons in fixed tissue sections and in dissociated cell cultures of rat primary visual cortex. SCP and $\mathrm{CP}$ neurons could be studied in the same preparation following in vivo injections of rhodamine- and fluorescein-conjugated latex beads (Katz et al., 1984; Katz and Iarovici, 1990) into the ipsilateral superior colliculus and the contralateral primary visual cortex, respectively. SCP and CP cells were labeled in single rat pups to verify that SCP and CP neurons are distinct cell populations (Catsmann-Berrevoets et al., 1980; Giffin et al., 1991) and, in addition, to allow us to compare the properties of cells isolated, maintained, and studied under identical conditions. The epifluorescence micrograph shown in Figure $1 A$ illustrates that following bead injections on P5, rhodamine bead-labeled SCP cell bodies (red) are restricted to layer 5 , whereas fluorescein beadlabeled CP cell somata (green) are present throughout cortical layers 2-6. Double-labeled cells were never detected during examinations of bead-labeled neurons either in tissue sections (Fig. $1 A, B$ ) or in dissociated cultures (Fig. $1 D$ ), indicating that SCP neurons and $\mathrm{CP}$ neurons are distinct, nonoverlapping populations of cortical projection neurons. 

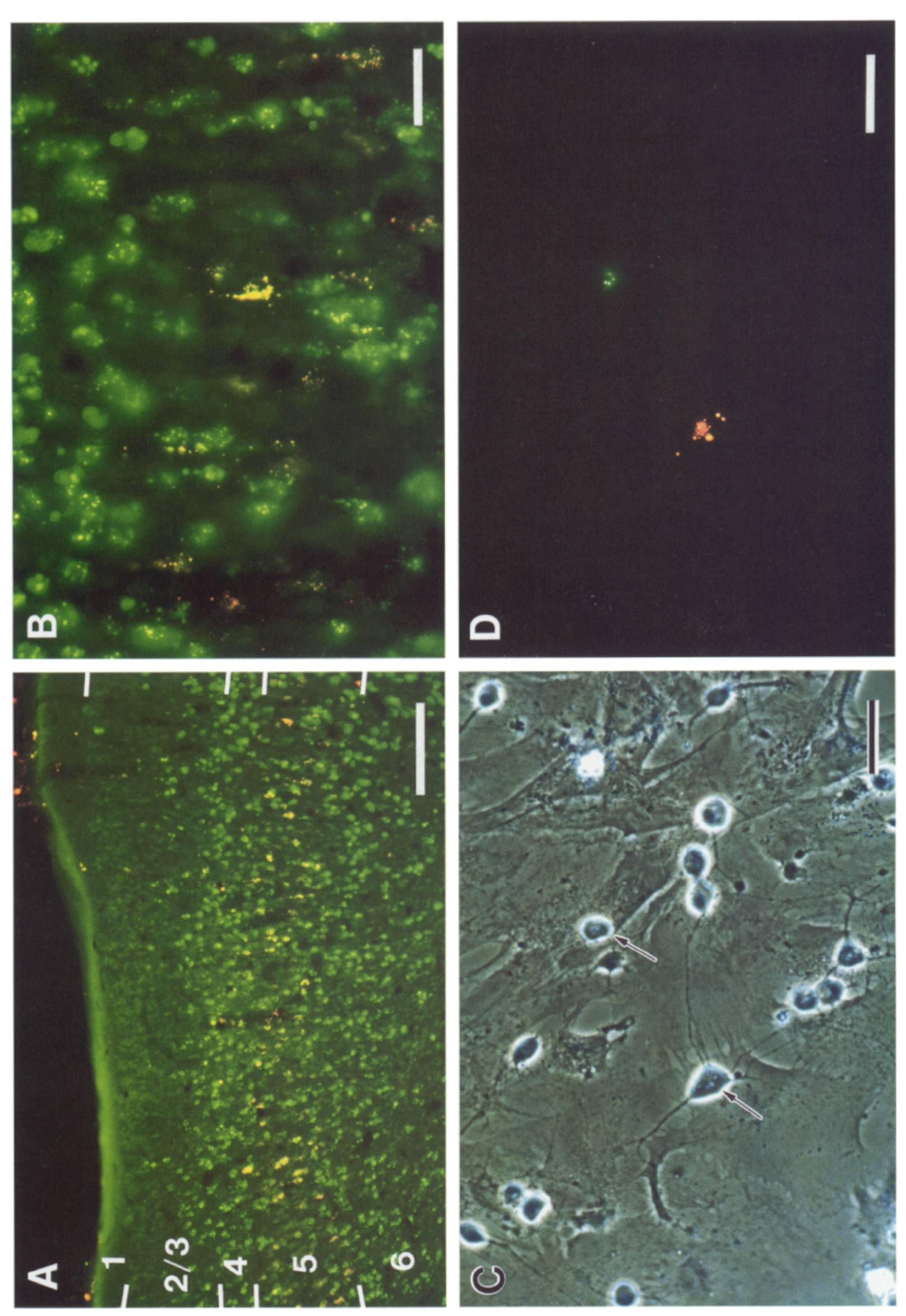

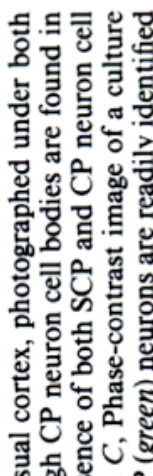

ตํㅓㅇ

굴

解记

旅

돈

勿

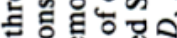

동ㅎㅁ

들

ญ它

है웡

呢

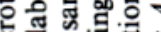

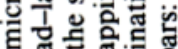

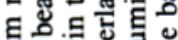

的布

잉

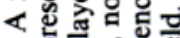

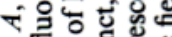

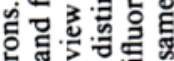

호등형

종.ำ

唯

응

혀웡

的里

बृँ

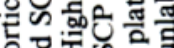
8 的

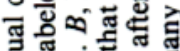
풍

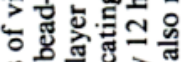
웅워웡 है है है 땡. 응돈 造 पू

대을

क्षे 댕요 च ष्ठ

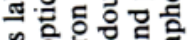
응 등 车论 댄듬 पू山

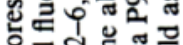

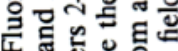
压

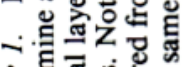

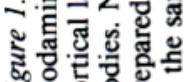

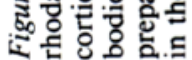




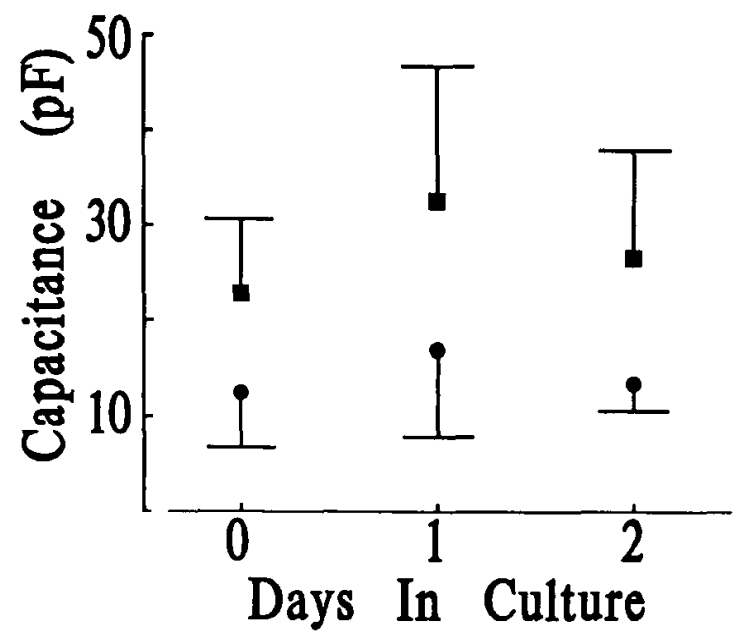

Figure 2. Cell capacitance of SCP and CP neurons over time in culture. Mean $( \pm$ SD) whole-cell capacitances of SCP $(\square)$ and $\mathrm{CP}(\bullet)$ neurons are plotted as a function of time in vitro. Note that, at all times, the mean capacitance of $C P$ cells was significantly lower than that of SCP neurons in the same preparations. For SCP cells, there was a measurable increase in mean cell capacitance between days 0 and 1 , but no further increase between days 1 and 2 was seen. No changes in mean CP cell capacitance as a function of time in vitro were observed (see Results).

\section{Electrophysiological recordings}

Hyperpolarization-activated inward currents were examined in whole-cell voltage-clamp recordings from 45 identified SCP neurons and 54 identified $\mathrm{CP}$ neurons in nine preparations. Cultures were prepared from neonatal rat pups on $\mathrm{P} 9, \mathrm{P} 12$, and P15 to allow examination of any possible age-dependent changes in current densities and properties over the second postnatal week. In addition, the possible effects of tissue dissociation and time in vitro were investigated by comparing recordings from freshly isolated cells with those from cells maintained for 1-2 $\mathrm{d}$ in culture. In Table 1, data from cells isolated from animals of various ages and data from cells maintained for varying times in vitro are provided. Although cell viability was high and recordings could readily be obtained from bead-labeled cells maintained for extended periods in vitro, detailed characterization of the electrical properties of such cells was limited because it was difficult to demonstrate adequate spatial control of the membrane voltage (see Materials and Methods). Even after only $1 \mathrm{~d}$ in vitro, many cells could not be clamped, and after $2 \mathrm{~d}$ in culture, voltage-clamp control could not be demonstrated in most SCP and CP neurons (Table 1).

\section{Cell capacitance}

Consistent with the anatomical data (Fig. 1), whole-cell membrane capacitance measurements, taken as a reflection of total cell membrane surface area and, therefore, cell size, suggest that SCP neurons represent a uniform cortical cell type, but that $\mathrm{CP}$ neurons are heterogeneous. We found, for example, that a histogram of SCP cell sizes is well described by a normal distribution ( $\chi^{2}$ test), whereas the distribution of CP cell sizes is not ( $\chi^{2}$ test, $p<0.0001$ ). No significant differences were observed for cells isolated on P9, P12, and P15 in either SCP (ANOVA) or CP (ANOVA and Kruskal-Wallis ANOVA by ranks) cells. SCP cell capacitance, however, was significantly greater than CP cell capacitance (Fig. 2) at all times examined ( $t$ test and Kolmogorov-Smirnov test, $p<0.01$ ). Although SCP cell capacitance increased over the first $24 \mathrm{hr}$ in vitro (ANOVA and Newman-Keuls tests), no further increases were evident between days 1 and 2 . This may reflect the facts that cells examined $2 \mathrm{~d}$ after isolation were selected for further analysis only if the decays of the capacitative transients were well described by single exponentials (see Materials and Methods) and the percentage of cells in which adequate spatial control of the membrane voltage could be demonstrated decreased dramatically between days 1 and 2 (Table 1). In contrast to the results with
SCP

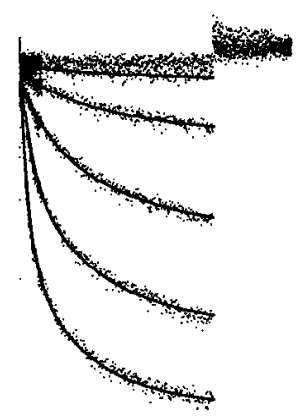

CP 1

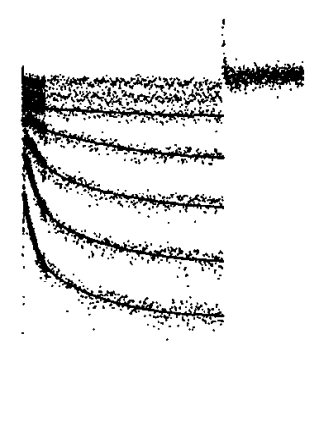

CP 2

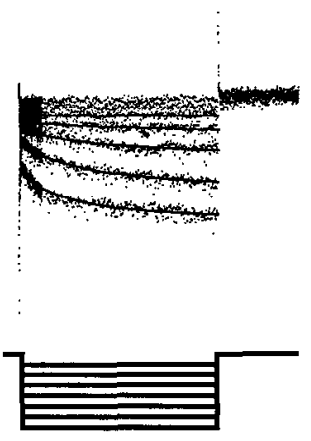

CP 3

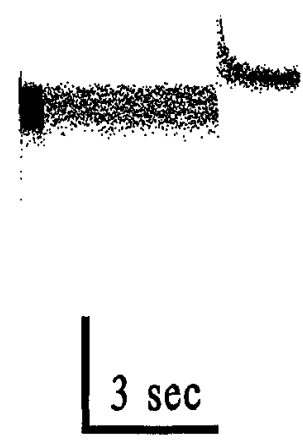

Figure 3. Variations in hyperpolarization-activated current waveforms in cortical projection neurons. Hyperpolarization-activated currents, recorded during voltage steps to potentials between -50 and $-110 \mathrm{mV}$ from a holding potential of $-40 \mathrm{mV}$, are plotted as points; the protocol is shown below the current records. Vertical calibration is $100 \mathrm{pA}$ for $S C P$ and $50 \mathrm{pA}$ for $C P 1-3$. Superimposed on the currents recorded during voltage steps to potentials negative to $-60 \mathrm{mV}$ in panels $S C P, C P 1$, and $C P 2$ are the best-fit solutions (lines) to Equation 1 with time constants from Table 2. SCP, Hyperpolarization-activated inward current waveforms recorded in a typical SCP neuron comprise three distinct components: a time-independent component $\left(I_{\text {inst }}\right)$, a slowly activating inward component $\left(I_{h, f}\right)$, and a very slowly activating inward component $\left(I_{h, s}\right)$. The two time-dependent components, which begin to activate between $-60 \mathrm{mV}$ and $-70 \mathrm{mV}$ and do not inactivate, are well described by Equation 1 . $C P$ $1-3$, Variations in hyperpolarization-activated current waveforms among CP neurons. $C P 1$, Hyperpolarization-activated inward current waveforms in the majority of CP neurons are similar to those seen in SCP neurons, but are lower in amplitude (note the change in scale). $C P$ 2, In a subset of CP cells, inward current waveforms appear to reflect only the presence of $I_{\text {inst }}$ and a single exponential corresponding to $I_{h . s}$ in Equation 1 . $C P$ 3, In a small number of CP cells, no time-dependent inward currents are evident on membrane hyperpolarizations (see also Fig. 5). 
A.

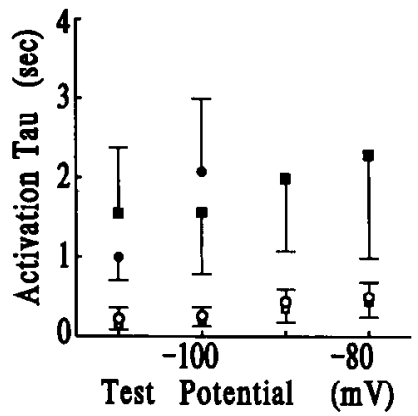

B.

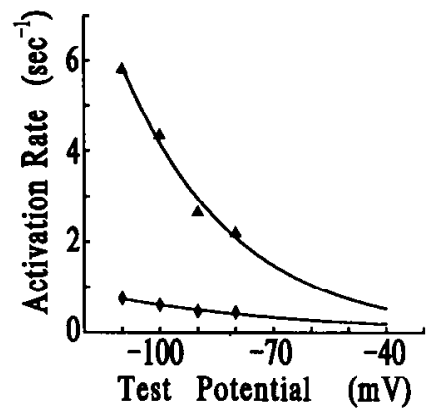

Figure 4. Activation time constants for $I_{h}$ and $I_{h}$, in SCP and CP neurons are indistinguishable. $A$, Activation time constants, determined by least-squares best fits of Equation 1 to hyperpolarization-activated inward current records, such as those illustrated in Figure 3, are plotted as a function of test potential for $\operatorname{SCP}(\square, \square)$ and $\operatorname{CP}(O, \bullet)$ neurons. Each point represents the mean of the values determined from at least 11 SCP neurons or at least five CP neurons. The error bars reflect thc SDs for the data from SCP neurons. $B$, Mean rate constants (equal to the reciprocals of the mean time constants), pooled from both SCP and $\mathrm{CP}$ neurons, are plotted as a function of test potential. Mean rate constants for $I_{h . s}(\Delta)$ and $I_{h . s}(\bullet)$ were least-squares fitted by the singleexponential expressions (Eq. 2) and are plotted here over the entire voltage range of activation (lines).

SĊP cells, no significant increases in whole-cell membrane capacitance were evident in CP cells maintained for $1-2 \mathrm{~d}$ in vitro (ANOVA and Kruskal-Wallis ANOVA).

\section{Distinct components of hyperpolarization-activated inward current}

The consistently observed response of SCP neurons hyperpolarized to potentials negative to $-70 \mathrm{mV}$ is an instantaneous increase in inward current followed by a slow, time-dependent inward current increase (Fig. 3, SCP). Similar inward current waveforms were recorded in the majority of $\mathrm{CP}$ cells examined (Fig. 3, CP 1), although the amplitudes of the currents were significantly lower in CP than in SCP cells (see also below). In addition, and in contrast to the similarity among SCP neurons, we observed considerable variability in the responses of $\mathrm{CP}$ neurons to hyperpolarizing voltage steps (Fig. 3, CP 1-3). In some $\mathrm{CP}$ cells, for example, distinct and very slowly activating inward currents were recorded (Fig. 3, CP 2) and, in others, no time-dependent inward currents were evident (Fig. 3, CP 3). The heterogeneity in the hyperpolarization-activated current waveforms among CP cells (Fig. 3) raised the interesting possibility that the hyperpolarization-activated current waveforms in $\mathrm{CP}$ and SCP cells may reflect the differential distribution of distinct conductance pathways. Subsequent analyses, therefore, were directed toward detailed characterization of the hyperpolarization-activated inward current waveforms in both cell types.

Recently, we demonstrated that the total hyperpolarizationactivated inward currents evoked in SCP neurons during prolonged voltage steps to potentials negative to $-60 \mathrm{mV}$ comprise three distinct components: (1) $I_{\text {inst }}$, an instantaneously activating, noninactivating current that may represent multiple resting conductance pathways; (2) $I_{h, s}$, a noninactivating inward current that activates over hundreds of milliseconds; and (3) $I_{h, s}$, a noninactivating inward current that activates over seconds (Solomon and Nerbonne, 1993a,b). Interestingly, neither $I_{h, f}$ nor $I_{h, s}$ in SCP cells inactivates measurably even during maintained

Table 2. Idealized activation time constants for $I_{h,}$ and $I_{h, s}$

\begin{tabular}{lll}
$\begin{array}{l}\text { Test potential } \\
(\mathrm{mV})\end{array}$ & $\tau_{h_{f}}(\mathrm{msec})$ & $\tau_{h, s}(\mathrm{msec})$ \\
\hline-50 & 1344 & 4415 \\
-60 & 952 & 3620 \\
-70 & 675 & 2969 \\
-80 & 478 & 2434 \\
-90 & 339 & 1996 \\
-100 & 240 & 1637 \\
-110 & 170 & 1342
\end{tabular}

Mean activation time constants for $I_{h, f}$ and $I_{h, s}$, derived from fits of Equation 1 to hyperpolarization-activated current records in both SCP and CP neurons. Current waveforms were fitted (as rate constants) to single-exponential expressions $(\mathrm{Eq}$ 2). Idealized activation time constants for $I_{h, s}$ and $I_{h, s}$ at each test potential were calculated from the corresponding rate constants on the two fitted curves (Fig. $4 B)$.

depolarizations (Solomon and Nerbonne, 1993b). Due to a voltage-dependent delay in the activation of $I_{h, f}$, biexponential expressions describing inward current activation in these cells are best fitted to the data when the $I_{\mathrm{h}, \mathrm{f}}$ term is raised to an exponent of 1.34 (Solomon and Nerbonne, 1993b). Inward current activation in SCP neurons is thus well described by the expression

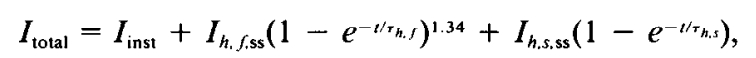

where $I_{\text {total }}$ is the total inward current, and $I_{h, x, s s}$ and $\tau_{h, x}$ are the steady state amplitudes and activation time constants, respectively, of the time-dependent inward current components. Time constants for inward current activation in SCP neurons, determined from fits to the rising phases of the currents recorded during membrane hyperpolarizations in records such as those illustrated in Figure 3, are plotted in Figure 4A. As noted above and illustrated in Figure 3 (CP 1) hyperpolarization-activated inward current waveforms in some CP neurons are similar to those in SCP neurons. For these cells, the currents were also well fitted by Equation 1, and the best least-squares fitted time constants for both $I_{h, f}$ and $I_{h, s}$ were not significantly different $(t$ test) from those in SCP cells (Fig. 4A). In addition, for both SCP and CP cells, neither $\tau_{h, f}$ nor $\tau_{h, s}$ varied as a function of animal age at isolation or length of time in culture.

Because of the small amplitudes of the hyperpolarizationactivated inward currents, the voltage range over which the time constants and the amplitudes of the individual current components could be determined was limited. This was particularly true for CP neurons in which the amplitudes of the hyperpolarization-activated currents were often very low. To determine the amplitudes and voltage dependences of $I_{i n s t}, I_{h, s}$, and $I_{h, s}$ in SCP and CP cells throughout the entire voltage range of activation, therefore, the voltage dependences of the activation rate constants of $I_{h, f}$ and $I_{h, s}$ were least-squares fitted to an exponential expression of the form

$$
k_{l, h, x}=A_{0} e^{A_{1} V},
$$

where $k_{l, h, x}$ is the activation rate constant of the current component $I_{h, x}\left(k_{l, h, x}=1 / \tau_{h, x}\right), V$ is the test potential, and $A_{0}$ and $A_{1}$ are free parameters. The mean activation rate constants for $I_{h, r}$ and $I_{h, s}$ determined from the pooled data from SCP and CP neurons were well described ( $r=0.99$ and $r=0.98$, respectively) by this expression (Fig. $4 B$ ). Values for $\tau_{\mathrm{h}, \mathrm{r}}$ and $\tau_{\mathrm{h}, \mathrm{s}}$ could then be estimated throughout the voltage range of inward current activation. The values are given in Table 2 . 
A.
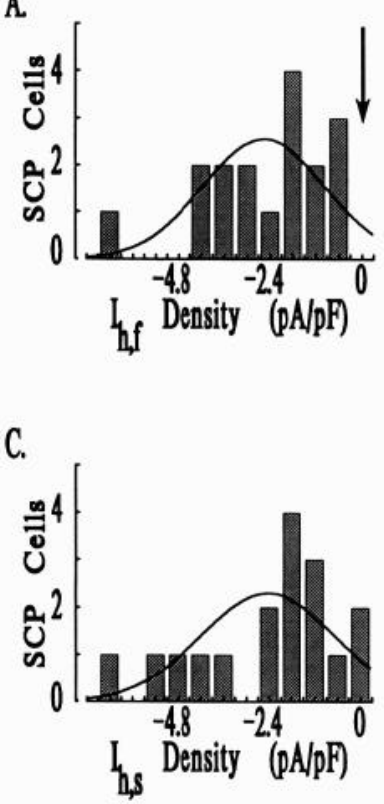

B.

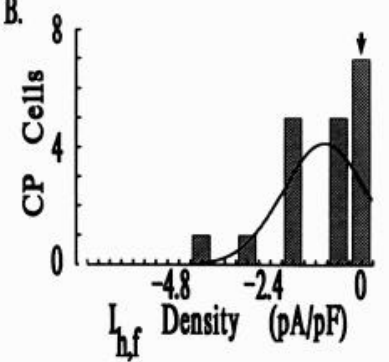

D.

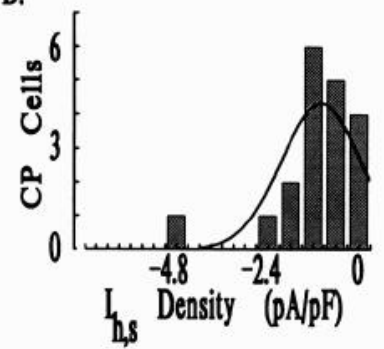

Figure 5. Densities of the time-dependent hyperpolarization-activated inward current components, $I_{h, f}$ and $I_{h . s}$, in SCP and CP neurons. $A-D$, Densities of $I_{h, s}$ and $I_{h, s}$ at $-100 \mathrm{mV}$ for cells studied on day 1 are plotted here as histograms; the bin width is $0.6 \mathrm{pA} / \mathrm{pF}$. Superimposed on the histograms are the best-fit normal distributions. $A$, The current density histogram for $I_{h, f}$ in SCP neurons is normally distributed with a mean of $-3 \mathrm{pA} / \mathrm{pf}$, and no cell had a density less than $0.6 \mathrm{pA} / \mathrm{pF}$ (arrow). B, The current density histogram for $I_{h, f}$ in CP neurons, in contrast, is not normally distributed, and seven cells are in the $0 \mathrm{pA}$ / $\mathrm{pF}$ bin (arrow). For both SCP $(C)$ and $\mathrm{CP}(D)$ cells, $I_{h, s}$ density histograms are normally distributed and, in both cell types, there are cells with no detectable $I_{h, s}$. Mean $I_{h, s}$ density, however, is lower in CP than in SCP neurons.

\section{Comparison of hyperpolarization-activated current components in SCP and CP neurons}

The amplitudes of $I_{\text {inst }}, I_{h, f}$, and $I_{h, s}$ were examined with respect to cell type (SCP or $\mathrm{CP}$ ), length of time in culture, and age at dissociation by fitting current records with Equation 1. As illustrated in Figure 3, the time-dependent inward current waveforms were well described by Equation 1 with time constants fixed to the values given in Table 2. Current amplitudes, determined from these fits, were normalized to cell capacitance and subsequently expressed as current densities to allow comparisons to be made among cells of differing sizes.

On visual inspection of the voltage-clamp data (see, e.g., Fig. 3), it appeared that both $I_{h, f}$ and $I_{h, s}$ were present in all SCP neurons, but that some CP cells displayed little or no detectable time-dependent inward currents. To determine if this impression was quantitatively correct, $I_{h, f}$ and $I_{h, s}$ amplitudes in individual cells were determined using Equation 1 and the time constants given in Table 2, and the densities of the two current components were calculated. The distributions of $I_{h, f}$ densities at $-100 \mathrm{mV}$ determined for 17 SCP cells and 19 CP cells examined at $1 \mathrm{~d}$ in vitro are tabulated in Figure $5 A-D$. Similar results were obtained for cells examined on day 2 (data not shown). As is evident in Figure 5A, $I_{h, f}$ is present in all SCP neurons, and the distribution of current densities is well described by a normal distribution. $I_{h . f}$ densities in CP neurons, in contrast, are not normally distributed $\left(\chi^{2}\right.$ test, $\left.p<0.05\right)$, and,

A.

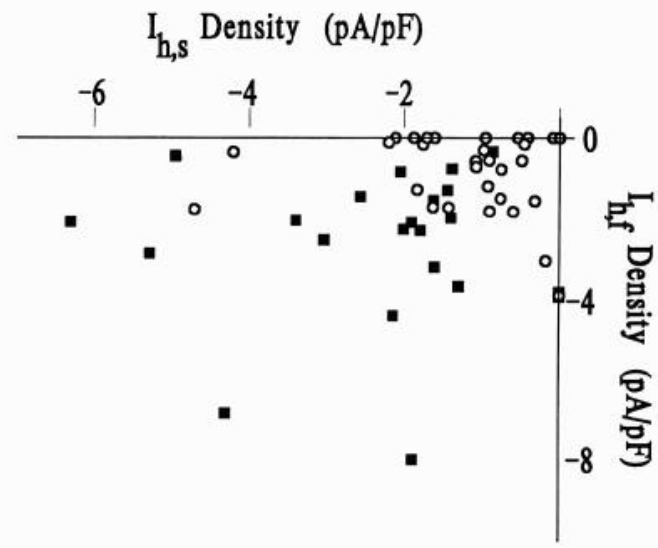

B.

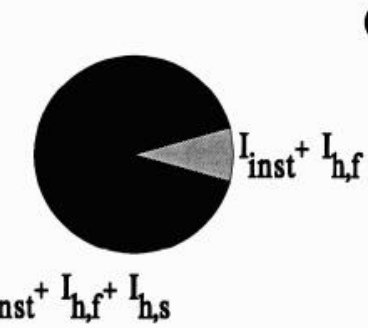

C.

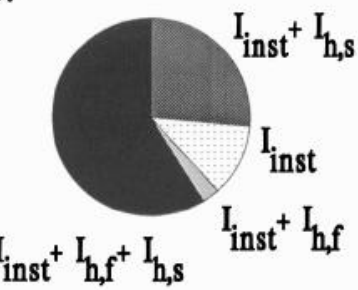

Figure 6. Comparison of $I_{h, f}$ and $I_{h, s}$ density distributions reveals distinct classes of cortical projection neurons. $A$, Plot of $I_{h, f}$ density versus $I_{h, s}$ density for all SCP ( $\square$ ) and CP (O) cells studied on days 1 and 2 reveals that the densities of both current components are substantially lower in CP than in SCP cells. Note that the point at the origin $(0,0)$ corresponds to the data from four cells. Interestingly, the distributions of current densities in CP neurons appeared to fall into groups, differing in the expression of one or both time-dependent inward current component(s). $B$ and $C$, Fractional representation of the distributions of hyperpolarization-activated inward currents in SCP and CP neurons. The density measurements in $A$ were grouped depending on the presence of $I_{\text {inst }}, I_{h, s}$, and $I_{h, s} . B$, For SCP neurons, both $I_{h, s}$ and $I_{h, s}$ were evident 21 of the 23 cells $(91 \%)$ examined; in the other two cells, only $I_{h . f}$ was detected (see Discussion). $C, \mathrm{CP}$ neurons, in contrast, could be partitioned into at least three distinct groups. In the majority of CP cells, both $I_{h, f}$ and $I_{h, s}$ were evident. In $26 \%$ of CP cells, however, only $I_{h,}$ was detected and, in $12 \%$ of the cells, no measurable time-dependent hyperpolarization-activated inward currents were recorded. There was also one CP cell in which only $I_{h, f}$ was present (see Discussion).

in 7 of the $19(37 \%)$ cells studied, no $I_{h, f}$ was detectable (Fig. $5 B$ ). Interestingly, $I_{h, s}$ densities at $-100 \mathrm{mV}$ are normally distributed in both SCP and CP neurons, although in two SCP and four CP cells, no measurable $I_{h, s}$ component was detected (Fig. $5 C, D$; see also below).

In Figure $6 A, I_{h, f}$ density is plotted versus $I_{h, s}$ density for the hyperpolarization-activated inward currents evoked at -100 $\mathrm{mV}$ in $23 \mathrm{SCP}$ and $34 \mathrm{CP}$ neurons. This plot suggested that $\mathrm{CP}$, but not SCP, neurons partition into groups that differ in terms of $I_{h, f}$ and $I_{h, s}$ expression. From the current density measurements, therefore, cells were grouped depending on the presence of $I_{\text {inst }}, I_{h, s}$, and $I_{h, s}$ (Fig. $6 A, B$ ). As is evident in Figure $6 A$, SCP neurons are quite homogeneous with respect to the expression of hyperpolarization-activated inward currents. Although 2 of the $23(8.7 \%)$ SCP cells appeared to lack $I_{h, s}$, these two cells likely reflect the low end of the normal distribution of $I_{h, s}$ densities (Fig. 5C). CP neurons, in contrast, appear to fall into (at least) three distinct groups (Fig. $6 \mathrm{C}$ ). In 20 of the 34 (or 59\%) $\mathrm{CP}$ cells examined, the waveforms of the hyperpolarization- 
A.

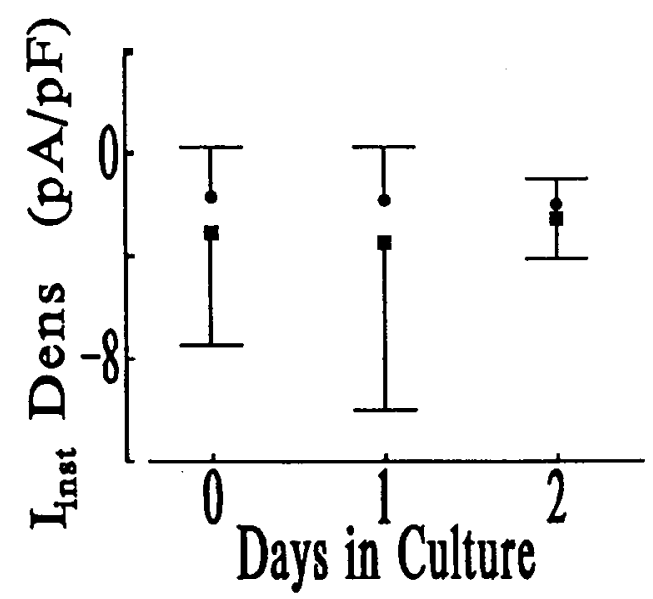

B.

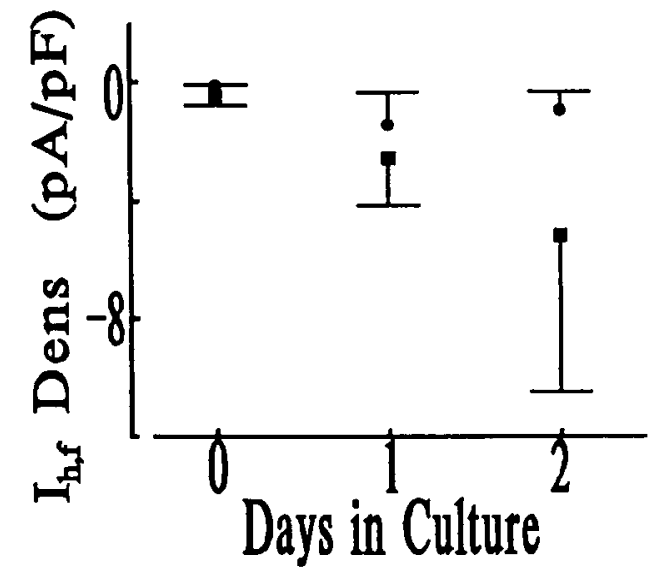

C.

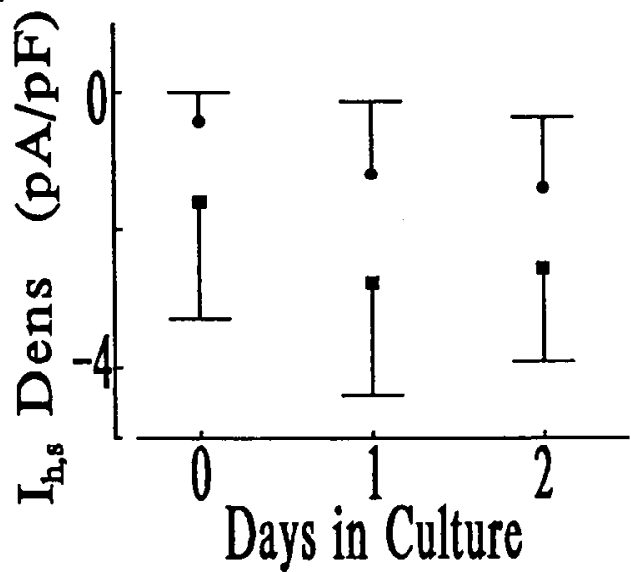

Figure 7. Variations in current densities as a function of time in culture. $A$, There were no significant changes in the densities of $I_{\text {inst }}$ over time in culture for either SCP (D) or CP (O) cells. The densities of $I_{h . f}$ $(B)$ and $I_{h, s}(C)$ increased significantly in both SCP $(D)$ and $C P(0)$ neurons between days 0 and 1 . Interestingly, there was also a further increase in $I_{h}$ density in SCP neurons $(B)$ between days 1 and 2, although $I_{h, s}$ density was not changed. In CP cells, neither $I_{h, f}$ nor $I_{h, s}$ density increased between days 1 and 2 . activated inward currents resemble those seen in SCP neurons, although, as noted above, current amplitudes are significantly lower in CP (Fig. 3, CP 1) than in SCP (Fig. 3, SCP) cells. As would be expected, analyses of the current waveforms revealed that both $I_{h, f}$ and $I_{h, s}$ are present in these cells. In 9 of the 34 (26\%) CP neurons (such as the cell illustrated in Fig. 3, CP 2), however, analyses of the inward current waveforms revcaled the presence of a single slowly activating inward current component; the hyperpolarization-activated currents in these cells are dominated by $I_{h, s}$. In Figure $6 A$, these are the nine cells clustered on the x-axis with $I_{h, f}$ density equal to zero. Finally, in 4 of the $34(12 \%) \mathrm{CP}$ cells, no time-dependent hyperpolarization-activated inward currents were evident (Fig. 3, CP 3); these 4 cells are plotted at the origin of Figure $6 A$. There was also one CP cell in which $I_{h, f}$ was present, but $I_{h, s}$ was not detectable. It is difficult to determine whether this represents a distinct class of CP neurons, or simply the low end of the distribution of $I_{h . s}$ densities in the subpopulation of CP neurons expressing both $I_{h, f}$ and $I_{h, s}$. By analogy, it might be suggested that cells displaying no time-dependent inward currents (Fig. 3, CP 3), in fact, represent the low end of the density distribution of cells displaying only $I_{h, s}$ at $-100 \mathrm{mV}$ (Fig. 3, CP 2). If this were the case, however, one would expect to find as many cells displaying only $I_{h, f}$ as cells displaying only $I_{\text {inst. }}$. Clearly, this was not found experimentally. Thus, it seems most likely that the apparent absence of $I_{h, s}$ in the two SCP cells and one CP cell in which $I_{h, f}$ was evident simply reflects the low end of the (normal) distributions of $I_{h, s}$ densities in these cells.

\section{Hyperpolarization-activated currents over time in culture}

Mean current densities at $-100 \mathrm{mV}$ for $I_{\text {inst }}, I_{h, f}$, and $I_{h, s}$ as a function of time in vitro are plotted in Figure 7; only nonzero current densities were included in the determinations of these means. As is evident, mean $I_{\text {inst }}$ density is not significantly different in SCP and CP cells and does not change over the first 2 $\mathrm{d}$ in culture (ANOVA). $I_{h, f}$ and $I_{h . s}$ densities, in contrast, increase significantly in both SCP and CP neurons (Kruskal-Wallis ANOVA) between days 0 and 2 in vitro. In addition, $I_{h, f}$ and $I_{h . s}$ densities are significantly larger in SCP neurons than in CP neurons over the first $50 \mathrm{hr}$ in culture ( $t$ test and KolmogorovSmirnov test, $p<0.05$ ). Differences in the densities of the timedependent currents between the two cell types were no longer significant by day 2 in vitro. Similar to the capacitance determinations described above, however, these measurements may well be influenced by the selection of neurons in which adequate spatial control of the membrane voltage could be demonstrated (see Materials and Methods). There were no differences in the densities of the hyperpolarization-activated current components in either SCP or CP cells isolated at P9, P12, or P15.

Voltage dependences of $\mathrm{I}_{\mathrm{h}, \mathrm{f}}$ and $\mathrm{I}_{\mathrm{h}, \mathrm{s}}$

The normalized current-voltage (I-V) relations for $I_{h, f}$ and $I_{h, s}$ are plotted in Figure 8. Normalized $I_{\text {inst }}$ was approximately linear in both SCP and CP neurons, intersecting the voltage axis at the average zero current potentials of -56 and $-58 \mathrm{mV}$ for $\mathrm{SCP}$ and $\mathrm{CP}$ cells, respectively (data not shown). The amplitudes of $I_{h . f}$ and $I_{h, s}$ in each cell were measured and normalized to their respective values recorded in the same cell at $-100 \mathrm{mV}$. In both cell types, $I_{h, f}$ begins to activate at potentials negative to $-60 \mathrm{mV}$ (Fig. $8 A$ ), and the normalized $I-V$ curves appear linear at potentials negative to approximately $-90 \mathrm{mV}$. In contrast to $I_{h . f}$, the normalized $I-V$ relations for $I_{h, s}$ in both SCP 
and CP neurons appear sigmoidal (Fig. $8 B$ ). Although $I_{h, s}$ also begins to activate negative to $-60 \mathrm{mV}$, the $I-V$ curve for $I_{h, s}$ is considerably steeper than that for $I_{h, f}$, and it appears to saturate (rather than become linear as seen for $I_{h, f}$ ) at potentials negative to approximately $-90 \mathrm{mV}$. A sigmoidal $I-V$ relationship may suggest complex ion permeation characteristics or atypical gating properties of the $I_{h . s}$ conductance pathway (see Discussion). There was no effect of age at dissociation or length of time in vitro on the voltage dependencies of $I_{h . f}$ or $I_{h, s}$ for either SCP or CP cells.

\section{Discussion}

\section{Distinct populations of cortical projection neurons}

Combining in vivo retrograde fluorescent labeling techniques and in vitro whole-cell recordings has allowed examination of the distribution of hyperpolarization-activated currents in two anatomically identified subpopulations of visual cortical projection neurons. Although both groups of cells were retrogradely labeled by virtue of their projections outside of area 17 , they are distinct cell populations. Consistent with previous doublelabeling studies (Catsmann-Berrevoets et al., 1980; Hallman et al., 1988), we found no cells labeled with both rhodamine- and fluorescein-conjugated beads following injections into the ipsilateral superior colliculus and the contralateral primary visual cortex. Thus, the two cell populations appear to be distinct and nonoverlapping.

SCP neurons are generally considered morphologically (Schofield et al., 1987) and anatomically (Hallman et al., 1988) homogeneous. Recent work suggests that SCP cells may also be electrophysiologically homogeneous (Mason and Larkman, 1990; Wang and McCormick, 1993). By visual inspection, rhodamine bead-labeled SC.P cells are among the largest cells in dissociated cortical cultures prepared from P9-P15 Long-Evans rat pups. The uniformly large size of these cells is also reflected in measurements of whole-cell membrane capacitances. SCP cell membrane capacitance was normally distributed and, assuming a specific membrane capacitance of $1 \mu \mathrm{F} / \mathrm{cm}^{2}$, our data suggest a mean diameter of approximately $26 \mu \mathrm{m}$ for SCP cells studied on the day of plating. The analyses of hyperpolarization-activated currents here also suggest that SCP neurons are rather uniform in that $I_{h, f}$ and $I_{h, s}$ densities are normally distributed, and $I_{h . f}$ is present in all cells. In 2 of the $23(9 \%)$ cells studied, however, no $I_{h, s}$ was detectable. Because $I_{h, s}$ amplitudes in these cells are small and $I_{h, s}$ densities are normally distributed; however, we suggest that the absence of $I_{h, s}$ likely reflects $I_{h, s}$ densities at the low end of the distribution, rather than that $I_{h . s}$ channels are not expressed in a subset (9\%) of SCP cells.

In contrast to SCP cells, CP neurons are generally considered to be a heterogeneous group of cortical neurons. CP cells, for example, display a variety of receptive field (Harvey, 1980) and ocular dominance (McCourt et al., 1990) properties. In addition, although the majority of CP cells are pyramidal, a substantial number are spiny stellate (Voigt et al., 1988). Recently, it has been reported that a number of smooth, nonpyramidal cells also project across the callosum (Buhl and Singer, 1989; Peters et al., 1990). In the experiments here, CP neurons were readily distinguished from SCP neurons by their passive and active membrane properties. As a group, CP neurons are significantly smaller than SCP cells, and the non-Gaussian distribution of cell capacitances suggests size heterogeneity within the population of CP cells. Heterogeneity among CP cells was also evident in recordings of hyperpolarization-activated currents. Cur-
A.

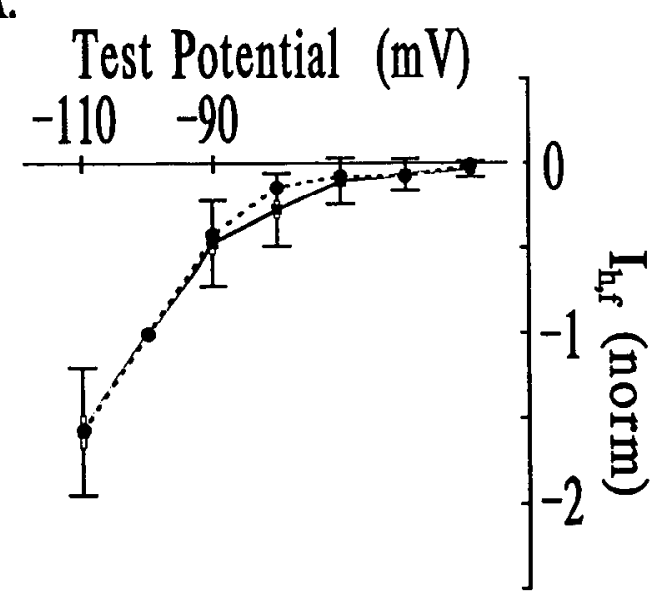

B.

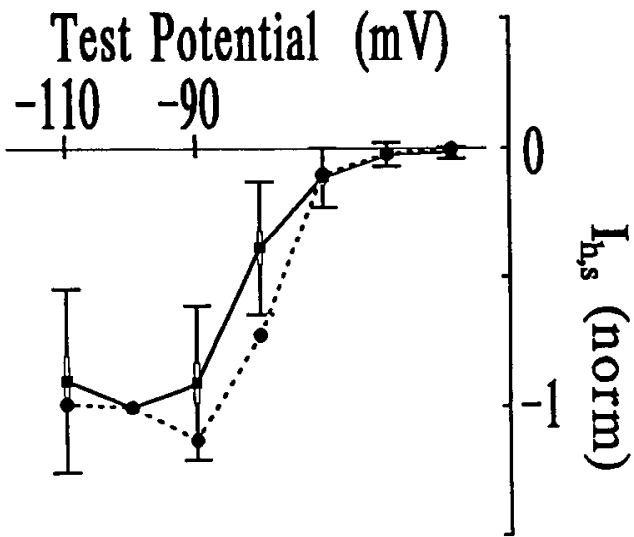

Figure 8. Voltage dependences of $I_{h .,}$ and $I_{h, .}, A$ and $B$, Normalized current-voltage $(I-V)$ relationships for $I_{h, f}(A)$ and $I_{h, s}(B)$ in $\operatorname{SCP}($ solid lines $)$ and $\mathrm{CP}(\boldsymbol{\theta}$, dashed lines) neurons examined on day 1 . For each cell, $I_{h, f}$ and $I_{h . s}$ amplitudes determined at each test potential were normalized to their respective amplitudes determined for the current recorded at $-100 \mathrm{mV}$. Mean normalized currents are plotted versus test potential. SEs (open boxes) and SDs (error bars) are shown for the measurements in SCP cells only. $A$, Normalized $I-V$ relations for $I_{h, f}$ in SCP and CP neurons are indistinguishable. The currents begin to activate between -60 and $-70 \mathrm{mV}$ and are nearly linear at potentials negative to $-90 \mathrm{mV}$. $B$, Normalized $I-V$ relations for $I_{h, s}$ in SCP and CP neurons are indistinguishable, although both are distinct from $I_{h . f}$. $I_{h, s}$ begins to activate at $-60 \mathrm{mV}$ and is steeply voltage dependent. The curves plateau at potentials negative to $-90 \mathrm{mV}$, thereby giving the overall $I-V$ curve a sigmoidal appearance (see Discussion).

rent density measurements partition CP neurons into three major classes: (1) cells with $I_{h, f}$ and $I_{h, s}(59 \%)$, (2) cells with $I_{h, s}$ only $(26 \%)$, and (3) cells with no measurable time-dependent hyperpolarization-activated inward currents (12\%). These results demonstrate that, in sharp contrast to our findings in SCP cells and in spite of the fact that all CP cells project to the same target, these cells are electrophysiologically quite heterogeneous. In addition, although $59 \%$ of the CP cells resemble SCP cells in that they express both $I_{h, f}$ and $I_{h, s}$, the densities of $I_{h, f}$ and $I_{h, s}$ in these cells are significantly lower than those measured in SCP neurons. Thus, although this subset of CP cells qualitatively resembles the entire population of SCP cells, quantitatively these 
cells are distinct. We should also note that because $I_{h, s}$ densities were normally distributed, it is possible that cells with no measurable time-dependent currents are a subset of cells that express only $I_{h, s}$ at undetectable levels. In addition, it is certainly possible that additional smaller subsets of $\mathrm{CP}$ cells exist.

\section{Changes over time in culture}

Electrical recordings were obtained from cells dissociated from rat pups between $\mathrm{P} 9$ and $\mathrm{P} 15$ and maintained in culture for up to $2.5 \mathrm{~d}$. Although age at isolation had no effect on any recorded parameter, length of time in culture had marked effects on capacitance and hyperpolarization-activated current measurements. Mean SCP cell capacitance increased significantly between days 0 and 1 . No further increase in capacitance was observed on day 2, although as noted previously, this likely results from selection of cells that could be voltage clamped. This selection tends to exclude cells with elaborate neurite outgrowth, which is robust in our cultures. Interestingly, no significant increase in mean CP cell capacitance was observed.

$I_{h, f}$ and $I_{h, s}$ densities increased in both SCP and CP neurons between days 0 and 1 . Because density measurements are normalized to cell capacitance and, thus, membrane surface area, an increase in current density over time in culture is not compatible with a model of cell growth in which homogeneous units of channel-containing membrane are added. In other words, unlike the expression of $I_{\text {inst }}$, the expression of time-dependent inward currents over time in culture is out of proportion with respect to the addition of new membrane. An increase in current density might be expected if time-dependent inward current channels were preferentially localized on cell processes. If neurite membrane contained a higher density of time-dependent inward current channels, the preferential growth of neurites with respect to the cell body would be reflected as an increase in current density. Alternatively, it is possible that the increases in $I_{h, f}$ and $I_{h, s}$ densities between days 0 and 1 in vitro reflect the preferential increase in time-dependent inward current channels in the cell bodies of CP and SCP cells. It is certainly also possible that both of these factors contribute. Further experiments will be necessary to clarify directly the underlying mechanisms.

It might be suggested that the finding of different classes of $\mathrm{CP}$ cells reflects the deleterious effects of the cell dissociation procedure on the hyperpolarization-activated inward currents in these cells. In our view, however, this seems highly unlikely for several reasons. First, and most importantly, the amplitudes and density distributions of hyperpolarization-activated currents in CP neurons are lower and more heterogeneous than those in SCP neurons derived from the same preparation. Second, membrane potential recordings from layer 5 neurons in the in vitro slice preparation reveal that the depolarizing sag in the membrane potential produced by $I_{h}$ activation is largest in layer 5 cells with the morphology of SCP cells, intermediate in layer 5 cells suspected to be CP cells, and undetectable in layer $2 / 3$ cells (Mason and Larkman, 1990). Finally, $I_{h, f}$ and $I_{h, s}$ densities in SCP and CP cells remain constant between days 1 and 2 in vitro. Thus, it seems highly unlikely that the finding of different $\mathrm{CP}$ classes reflects some artifact of the tissue dissociation and culture methodologies. Rather, we interpret the finding that $I_{h, f}$ and $I_{h, s}$ are differentially expressed in CP cells as indicating that these two current components reflect the presence of two functionally distinct conductance pathways or channels that are differentially distributed in subtypes of CP cells.
Distinct properties of $\mathrm{I}_{\mathrm{h}, \mathrm{s}}$ and $\mathrm{I}_{\mathrm{h}, \mathrm{s}}$

The time- and voltage-dependent properties of $I_{h, f}$ and $I_{h, s}$ in CP neurons are indistinguishable from those in SCP neurons (Solomon and Nerbonne, 1993b). The normalized $I-V$ relationship for $I_{h, f}$ is also similar to that previously reported for the total hypcrpolarization-activated current in SCP neurons (Solomon and Nerbonne, 1993a). Interestingly, however, the normalized $I-V$ relations for $I_{h, s}$ in both cell types are quite different from that of $I_{h, f}$. Although both $I_{h, f}$ and $I_{h, s}$ begin to activate at approximately $-60 \mathrm{mV}$, the activation of $I_{h, s}$ is more steeply voltage dependent and saturates near $-90 \mathrm{mV}$. Saturation of the $I_{h, s} I-V$ curve indicates that, in the face of an increasing driving force, there is a decrease in $h, s$ conductance. Although it is possible that the saturation of the $I-V$ curves results from incorrect current amplitude measurements derived from a poorly fitting kinetic model, this explanation seems unlikely because the time constants used to determine the current amplitudes in the range of voltages where saturation was observed are not on the extrapolated portion of the model (Eq. 2). Saturation also likely does not reflect difficulties in resolving $I_{h, s}$ from the baseline $\left(I_{\text {inst }}\right)$ or from $I_{h, s}$ because saturation of $I_{h, s}$ occurs at extremely hyperpolarized potentials where the activation time constants for $I_{h, s}$ are approximately 3 orders of magnitude larger than those of $I_{h, f}$, and the currents are most readily distinguished from $I_{\text {inst }}$. Rather, it seems most likely that the sigmoidal $I-V$ relation for $I_{h, s}$ reflects unconventional aspects of the channel gating or ion permeation properties of $I_{h, s}$ channels. In a simple two state model, a biphasic $I-V$ relationship could be generated if the unidirectional ratc constant for channel closing is more steeply voltage dependent than the rate constant for channel opening. In this case, although the activation time constants might continue to decrease with hyperpolarization, the conductance would also start to decrease as the total number of open channels begins to be controlled by the closing rate. A second possibility is that the conductance may decrease with increasing driving force due to voltage-dependent open channel block. Further experiments will be necessary to distinguish between these possibilities.

\section{Physiological roles for hyperpolarization-activated currents}

The time-dependent hyperpolarization-activated currents in SCP neurons have been shown to underlie the depolarizing sag and overshoot phenomena observed during and following, respectively, hyperpolarizing current injections (Solomon and Nerbonne, 1993a). Because of the very slow kinetics of activation and deactivation of these currents (Solomon and Nerbonne, 1993b), these changes may represent a type of electrical "shortterm memory." That is, because of the presence of $I_{h}$ channels, sustained or repetitive hyperpolarizing inputs could alter cell resting membrane potential and input resistance over a time course of hundreds of milliseconds. Also, because the posthyperpolarization overshoot of the resting membrane potential can be of sufficient magnitude to bring a cell to threshold (Solomon and Nerbonne, 1993a), the activation of these time-dependent inward currents may be important in generating patterns of posthyperpolarization "rebound" firing (Spain et al., 1991). It is important to note that, although the experiments here have focused on the properties and densities of the $I_{h}$ channels expressed in or near the cell bodies of CP and SCP cells, the presence of $I_{h}$ channels at sites distant from the soma is also expected to be important in determining the responses to syn- 
aptic inputs and in determining the overall input-output relations of different types cortical neurons.

The physiological significance of the variations in hyperpolarization current densities between SCP and CP neurons, as well as among the different classes of CP neurons, remains to be determined. Interestingly, it has been demonstrated that $\mathrm{CP}$ cells receive dense GABAergic innervation, and that the number of inhibitory contacts on CP cells is higher than on geniculateprojecting cells (Fariñas and DeFelipe, 1991). Relating these findings to the results presented here points to the importance of understanding the intrinsic membrane properties of cortical neurons, in addition to the synaptic connections between neurons, if one really hopes to understand how cortical circuits, as well as each of the individual ncurons within the circuit, function. For example, in spite of marked differences in the numbers of inhibitory synaptic contacts made on SCP and CP cells, it is possible that the two cell types will respond in a similar fashion to synaptic inhibition due to the differential expression of $I_{h}$ channels. Alternatively, differences in the densities of $I_{h, f}$ and $I_{h, s}$ among CP cells would be expected to influence how different (CP) cells respond to the onset and offset of synaptic inhibition, even if the number of inhibitory contacts on each cell is the same. It will be of considerable interest to examine the role of $I_{\text {inst }}, I_{h, f}$, and $I_{h, s}$ in determining the responses of different types of cortical projection neurons to inhibitory, as well as excitatory, synaptic inputs.

\section{References}

Buhl EH, Singer W (1989) The callosal projection in cat visual cortex as revealed by a combination of retrograde tracing and intracellular injection. Exp Brain Res 75:470-476.

Catsmann-Berrevoets CE, Lemon RN, Verburgh CA, Bentivoglio $M$, Kuypers HGJM (1980) Absence of callosal collaterals derived from rat corticospinal neurons. Exp Brain Res 39:433-440.

Chagnac-Amitai Y, Luhmann HJ, Prince DA (1990) Burst gencrating and regular spiking layer 5 pyramidal neurons of rat neocortex have different morphological features. J Comp Neurol 296:598-613.

Connors BW, Gutnick MJ (1990) Intrinsic firing patterns of diverse neocortical neurons. Trends Neurosci 13:99-104.

DiFrancesco D (1981) A study of the ionic nature of the pacemaker current in calf Purkinje fibres. J Physiol (Lond) 314:377-393.

Fariñas I, DeFelipe J (1991) Patterns of synaptic input on corticocortical and corticothalamic cells in the cat visual cortex. 1 . The cell body. J Comp Neurol 304:53-69.

Giffin K, Solomon JS, Burkhalter A, Nerbonne JM (1991) Differential expression of voltage-gated calcium channels in identified visual cortical neurons. Neuron 6:321-332.

Hallman ER, Schofield BR, Lin C-S (1988) Dendritic morphology and axon collaterals of corticotectal, corticopontine, and callosal neurons in layer $\mathrm{V}$ of primary visual cortex of the hooded rat. J Comp Neurol 272:149-160.

Hamill OP, Marty A, Neher E, Sakmann B, Sigworth FJ (1981) Improved patch-clamp techniques for high resolution current recording from cells and cell-free membrane patches. Pfluegers Arch 391:85100.

Harvey AR (1980) A physiological analysis of subcortical and commissural projections of areas 17 and 18 of the cat. J Physiol (Lond) 302:507-534.

Huettner JE, Baughman RW (1986) Primary culture of identified neurons from the visual cortex of postnatal rats. J Neurosci 6:3044-3060.

Innocenti GM (1981) Growth and reshaping of axons in the establishment of visual callosal connections. Science 212:824-827.

Ivy GO, Akers RM, Killackey HP (1979) Differential distribution of callosal projection neurons in the neonatal and adult rat. Brain Res 173:532-537.

Katz LC, Iarovici DM (1990) Green fluorescent latex microspheres: a new retrograde tracer. Neuroscience 34:511-520.

Katz LC, Burkhalter A, Dreyer WJ (1984) Fluorescent latex microspheres as a retrograde neuronal marker for in vivo and in vitro studies of visual cortex. Nature 310:498-500.

Mason A, Larkman A (1990) Correlations between morphology and electrophysiology of pyramidal neurons in slices of rat visual cortex. II. Electrophysiology. J Neurosci 10:1415-1428.

McCormick DA, Connors BW, Lighthall JW, Prince DA (1985) Comparative electrophysiology of pyramidal and sparsely spiny stellate neurons of the neocortex. J Neurophysiol 54:782-806.

McCourt ME, Thalluri J, Henry GH (1990) Properties of the area 17/ 18 border neurons contributing to the visual transcallosal pathway in the cat. Vis Neurosci 5:83-98.

Peters A, Payne BR, Josephson K (1990) Transcallosal non-pyramidal cell projections from visual cortex in the cat. J Comp Neurol 302: 124-142.

RaffMC, Fields KL, Hakomori S, Mirsky R, Pruss RM, Winter J (1979) Cell type-specific markers for distinguishing and studying neurons and the major classes of glial cells in culture. Brain Res 174:283-308.

Schofield BR, Hallman LE, Lin C-S (1987) Morphology of corticotectal cells in the primary visual cortex of hooded rats. J Comp Neurol 261:85-97.

Solomon JS, Nerbonne JM (1993a) Hyperpolarization-activated currents in isolated superior colliculus-projecting neurons from rat visual cortex. J Physiol (Lond) 462:393-420.

Solomon JS, Nerbonne JM (1993b) Two kinetically distinct components of hyperpolarization-activated current in rat superior colliculusprojecting neurons. J Physiol (Lond), in press.

Spain WJ, Schwindt PC, Crill WE (1991) Post-inhibitory excitation and inhibition in layer $\mathrm{V}$ pyramidal neurones from cat sensorimotor cortex. J Physiol (Lond) 434:609-626.

Thong IB, Dreher B (1986) The development of the corticotectal pathway in the albino rat. Brain Res 390:227-238.

Voigt T, LeVay S, Stamnes MA (1988) Morphological and immunocytochemical observations on the visual callosal projections in the cat. J Comp Neurol 272:450-460.

Wang Z, McCormick DA (1993) Control of firing mode of corticotectal and corticopontine layer $\mathrm{V}$ burst-generating neurons by norepinephrine, acetylcholinc, and IS,3R-ACPD. J Neurosci 13:2199-2216. 